\title{
Estadísticos poblacionales de Triatoma sordida Stäl 1859 (Hemiptera : Reduviidae) en condiciones experimentales*
}

\section{Population statistics of Triatoma sordida Stäl 1859 (Hemiptera: Reduviidae) in experimental conditions}

\author{
Elena B. Oscherov, Miryam P. Damborsky, María E. Bar y Eduardo Porcel \\ Cátedra de Artrópodos de la Facultad de Ciencias Exactas y Naturales y Agrimensura de la \\ Universidad Nacional del Nordeste. Corrientes, Argentina
}

\begin{abstract}
Resumen
Se realizó el seguimiento longitudinal de cuatro cohortes de 100 huevos cada una. Los insectos fueron criados a $28^{\circ} \mathrm{C} \pm 3^{\circ} \mathrm{C}$ y $63 \% \pm 10 \%$ de humedad relativa, y alimentados cada siete días sobre gallina (Gallus domesticus), durante $40 \mathrm{~min}$. Las cohortes fueron controladas una vez por semana. Se observó un valor constante de supervivencia en las cuatro cohortes, sin picos importantes. La expectativa de vida de los machos, en promedio, fue de 37,4 semanas y la de las hembras 36,8 . El tiempo generacional demandó 61,7 semanas. La tasa reproductiva neta indica que esta población se incrementará 143,3 veces con cada generación. La tasa intrínseca de crecimiento natural fue de 0,082 por semana. El máximo valor reproductivo se verificó, en promedio, a la tercer semana del ingreso al estado adulto. La distribución estable de edades de $T$. sordida sería de $32 \%$ huevos, $26 \%$ ninfas de primer estadio, $19 \%$ ninfas de segundo estadio, $13 \%$ ninfas de tercer estadio, $6 \%$ ninfas de cuarto estadio, $3 \%$ ninfas de quinto estadio y $1 \%$ de adultos. Es la primera cita para Argentina sobre la tabla de vida de $T$. sordida. El análisis de estos parámetros permite considerar que esta especie se comporta como "K estratega", en condiciones experimentales.
\end{abstract}

Triatoma. Insectos vectores.

\begin{abstract}
Four cohorts of 100 Triatoma sordida eggs were reared under standard laboratory conditions $\left(28^{\circ} \mathrm{C}, 63 \%\right.$ R.H.) and fed weekly on hens during 40 minutes. The numbers of dead insects, moults and eggs laid were recorded weekly. A constant survival value was observed without any important peaks in the four cohorts. Life expectancy was 37.4 weeks for males and 36.8 weeks for females. The average generation time was 61.7 weeks. The average net reproduction rate suggests that this population would increase 143.2 times during each generation. The average intrinsic rate of natural increase was 0.082 per individual per week. The highest reproductive value was observed 3
\end{abstract}

\footnotetext{
*Investigación subsidiada por el Consejo Nacional de Investigaciones Científicas y Técnicas/SECYT-UNNE (Resolución n ${ }^{2305 / 87-259)}$ Correspondencia para/Correspondence to: Elena B. Oscherov - Cátedra de Artrópodos de la Facultad de Ciencias Exactas y Naturales y Agrimensura de la Universidad Nacional del Nordeste. 9 de Julio 1449. 3400 Corrientes, Argentina

Recebido en 25.9.1995. Reapresentado en 27.2.1996. Aprobado en 2.4.1996.
} 
weeks after entering the adult stage. The age structure, assuming stable age distribution, would be $32 \%$ eggs, $26 \%$ of first instar nymphs, $19 \%$ of second instar nymphs, $13 \%$ of third instar nymphs, $6 \%$ of fourth instar nymphs, $3 \%$ of fifth instar nymphs and $1 \%$ of adults. Our results suggest that this species behaves as a $k$-strategist under experimental conditions.

Triatoma. Insect vectors.

\section{Resumo}

Foram estudados diferentes parâmetros populacionais de Triatoma sordida em condições de laboratório. Realizou-se um seguimento longitudinal de quatro coortes de 100 ovos cada uma; os insetos foram criados a $28^{\circ} \mathrm{C} \pm 3^{\circ} \mathrm{C}$ e $63 \% \pm$ $10 \%$ de umidade relativa, e alimentados cada sete dias sobre galinha (Gallus domesticus) durante 40'. As coortes foram controladas uma vez por semana. A expectativa de vida média dos machos foi de 37,4 semanas e a das fêmeas de 36,8. O tempo médio de desenvolvimento foi de 61,7 semanas. A taxa reprodutiva líquida indica que esta população aumentará 143,2 vezes durante cada geração. A taxa intrínseca do crecimento natural foi de 0,082 por semana. A média do valor máximo reprodutivo verificou-se na terceira semana no início do estádio adulto. A distribuição estável de idades de T. sordida seria de $32 \%$ ovos, $26 \%$ ninfas do primeiro estádio, $19 \%$ ninfas do segundo estádio, $13 \%$ ninfas do terceiro estádio, $6 \%$ ninfas do quarto estádio, $3 \%$ ninfas do quinto estádio e $1 \%$ de adultos. A análise dos parâmetros permite considerar que esta espécie comporta-se como K-estrategista em condições experimentais.

\section{Triatoma. Insetos vetores.}

\section{INTRODUCCIÓN}

Triatoma sordida Stäl, 1859 es señalada como vector secundario del Trypanosoma cruzi, agente etiológico de la enfermedad de Chagas ${ }^{5,6,16}$. Su amplitud ecológica le permite colonizar una variedad de hábitats silvestres y domésticos ${ }^{1,2,4,14,16}$. Las aves son su principal fuente de alimento, pero también se nutre a expensas de mamíferos tales como el hombre, perro, gato y roedores ${ }^{5,6,8}$.

Desde el punto de vista epidemiológico es importante conocer las características biológicas de los triatominos, y una manera de evaluarlas es mediante la confección de tablas de vida. Al respecto, caben citarse los trabajos de Rabinovich ${ }^{13}$ sobre estadísticas vitales de T. infestans y los de Ghilini ${ }^{9,10}$ referidos a T. guasayana y T. breyeri. En la Argentina, aún no se han realizado estudios de este tipo con T. sordida, por lo que se consideró de sumo interés la confección de las tablas de vida y fecundidad de esta especie.

En lo presente trabajo se presentan los resultados obtenidos en el seguimiento de cuatro cohortes de T. sordida que aportan conocimientos sobre parámetros poblacionales tales como la esperanza de vida, el tiempo generacional, la tasa neta de reproducción, la tasa intrínseca de crecimiento natural y el valor reproductivo.

\section{MATERIAL Y MÉTODO}

El material biológico fue obtenido a partir de hembras provenientes del palomar situado en un parque público de la ciudad de Corrientes, Argentina.

Se conformaron cuatro cohortes de 100 huevos cada una $\left(\mathrm{N}_{0}\right)$, ovipuestos en el lapso de $24 \mathrm{~h}$. Los ejemplares fueron mantenidos en recipientes de plástico acondicionados con papel secante plegado en abanico, para facilitar el acceso a la fuente de alimento y absorber el exceso de humedad. Los machos y hembras, formando parejas, fueron separados de las ninfas, para el mejor control de la fecundidad.

La experiencia se llevó a cabo bajo condiciones controladas de temperatura $\left(28^{\circ} \mathrm{C} \pm 3^{\circ} \mathrm{C}\right)$ y humedad relativa $(63 \% \pm 10 \%$ H.R.). El fotoperíodo fue el correspondiente a la latitud de la ciudad de Corrientes. Los ejemplares fueron alimentados semanalmente sobre gallina, durante 40 minutos.

La duración del estudio, hasta que murió el último ejemplar, abarcó 41 meses. Una vez por semana se registraron los datos sobre mortalidad y fecundidad. Las fórmulas utilizadas para obtener los estadísticos vitales ${ }^{12,13}$ son las especificadas a continuación:

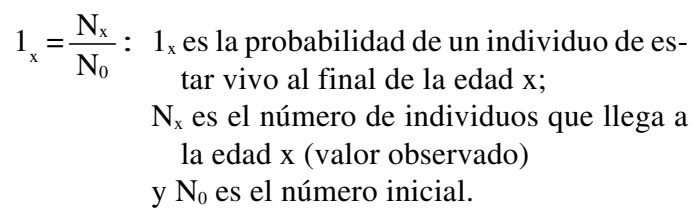



$\mathrm{T}_{\mathrm{x}}=\sum_{\mathrm{x}=\varnothing}^{\mathrm{w}} \quad \begin{aligned} & \mathrm{Lx}: \\ & \quad \begin{array}{l}\text { número total de semanas vividas } \\ \text { por la cohorte, desde } \mathrm{x} \text { hasta que }\end{array}\end{aligned}$ todos mueren $\mathrm{L}_{\mathrm{x}}=\left(1_{\mathrm{x}}+1_{\mathrm{x}+1}\right) / 2$

$\mathrm{e}_{\mathrm{x}} \quad($ expectativa de vida $)=\mathrm{T}_{\mathrm{x}} / \mathrm{l}_{\mathrm{x}}$

$\mathrm{m}_{\mathrm{x}}$ (fecundidad específica para la edad $\mathrm{x}$ )

$\mathrm{m}_{\mathrm{x}}$ (fecundidad específica para la edad $\mathrm{x}$, corregida por la proporción de sexos)

$\mathrm{T}($ tiempo generacional $)=\sum_{\mathrm{x}=\alpha}^{\mathrm{w}} \mathrm{x} 1_{\mathrm{x}} \mathrm{m}_{\mathrm{x}}^{\prime}$

TRB (tasa reproductiva bruta) $=\sum \mathrm{m}_{\mathrm{x}}^{\prime}$

$\mathrm{R}_{0}$ (tasa de reproducción neta) $=\sum_{\mathrm{x}=\alpha}^{\mathrm{w}} 1_{\mathrm{x}} \mathrm{m}_{\mathrm{x}}^{\prime}$

$\alpha$ es la edad de la primera reproducción y w es la edad de la última reprodución (valores observados).

$\mathrm{r}$ (tasa intrínseca de incremento natural): es el valor que satisface la ecuación $\sum_{x=\alpha}^{w} 1_{x} m_{x} e^{-r x}=1$

$\lambda$ (tasa finita de incremento natural) $\mathrm{e}^{\mathrm{r}}$

$\mathrm{V}_{\mathrm{x}}($ valor reproductivo $)=\frac{\mathrm{e}^{\mathrm{rx}}}{1_{\mathrm{x}}} \sum_{\mathrm{y}=\mathrm{x}}^{\infty} \mathrm{e}^{-\mathrm{ry}} 1_{\mathrm{y}} \mathrm{m}_{\mathrm{y}}$

DEE ( distribución estable de edades) $=$

$=\frac{\mathrm{L}_{\mathrm{x}} \mathrm{e}^{\mathrm{r}(\mathrm{x}+1)}}{\sum 1_{\mathrm{x}} \mathrm{e}^{\mathrm{r}(\mathrm{x}+1)}} \times 100$

\section{RESULTADOS}

\section{Supervivencia y Expectativa de Vida}

El número de sobrevivientes decreció en forma paulatina en las cuatro cohortes (Figura 1). En la cohorte A la mortalidad se acentuó en la semana 45, la que corresponde a la edad media en que las ninfas de esta cohorte ingresaron al estado adulto, y en la cohorte D en las semanas 2, 20 y 79.
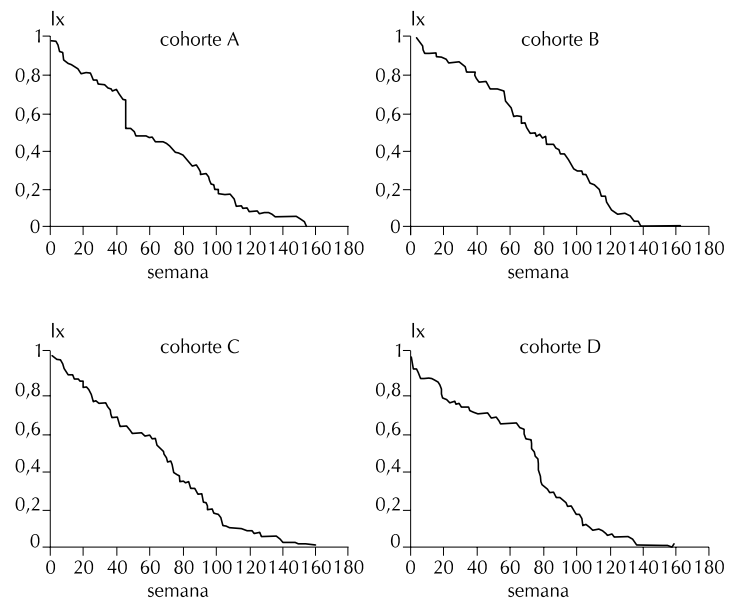

Figura 1 - Curvas de supervivencia de las cuatro cohortes de Triatoma sordida.
Respecto a la expectativa de vida, el aumento verificado en las ninfas de primer estadio de las cohortes A y D, reflejan la mortalidad producida en el estado de huevo. Al comenzar el estado adulto, los machos tuvieron una expectativa de vida media (37,4 semanas) semejante a la de las hembras (36,8 semanas) $(t=0,19 \mathrm{p}=0,86)$. Solamente en la cohorte $\mathrm{A}$ las hembras presentaron una expectativa de vida $(36,1$ semanas) superior a la de los machos (31,3 semanas) (Tabla 1).

Tabla 1 - Expectativa de vida de triatoma sordida (al ingresar a cada edad) bajo condiciones controladas de temperatura $\left(28^{\circ} \mathrm{C} \pm 3^{\circ} \mathrm{C}\right)$ y humedad relativa $(63 \% \pm$ $10 \%)$.

\begin{tabular}{lcccccc}
\hline \multicolumn{7}{c}{ Cohortes } \\
\hline Estadio & A & B & C & D & $\bar{X}$ & S \\
\hline Huevo & 62,6 & 75,9 & 64,8 & 66,9 & 67,5 & 5,8 \\
N1 & 63,5 & 74,9 & 62,8 & 69,7 & 67,7 & 5,7 \\
N2 & 62,8 & 72,4 & 60,7 & 67,9 & 65,9 & 5,3 \\
N3 & 62,0 & 73,1 & 61,3 & 65,5 & 65,5 & 4,7 \\
N4 & 57,8 & 66,4 & 56,3 & 59,9 & 60,1 & 4,4 \\
N5 & 51,5 & 55,5 & 53,8 & 60,4 & 55,3 & 3,8 \\
Hembras & 36,1 & 34,5 & 33,2 & 43,3 & 36,8 & 4,5 \\
Machos & 31,3 & 34,5 & 39,7 & 44,3 & 37,4 & 5,7 \\
\hline
\end{tabular}

Dado que la supervivencia fue decreciendo con la edad sin que se registraran picos importantes, la expectativa de vida también disminuyó en forma correlativa (Figura 2). Se observó un pequeño aumento de $e_{x}$ en la octava y novena semana (debido a que la mortalidad de las ninfas de segundo estadio se concentró en este período), y también al comienzo de la edad adulta y a partir de la semana 84 (previa a la edad media de mortalidad de los imagos) (Figura 2).

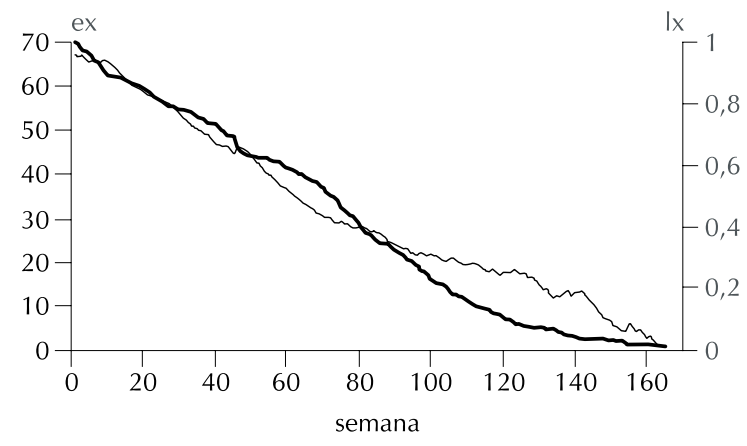

Figura 2- Curvas de supervivencia y expectativa de vida de Triatoma sordida. 


\section{Reproducción}

Las primeras oviposturas de las hembras de las cohortes A, B, C y D se produjeron en las semanas de vida 45,$8 ; 56,0 ; 41,7$ y 45,0 respectivamente $\overline{(X}=47,2 ; S=6,2 ; C V=13,1)$.
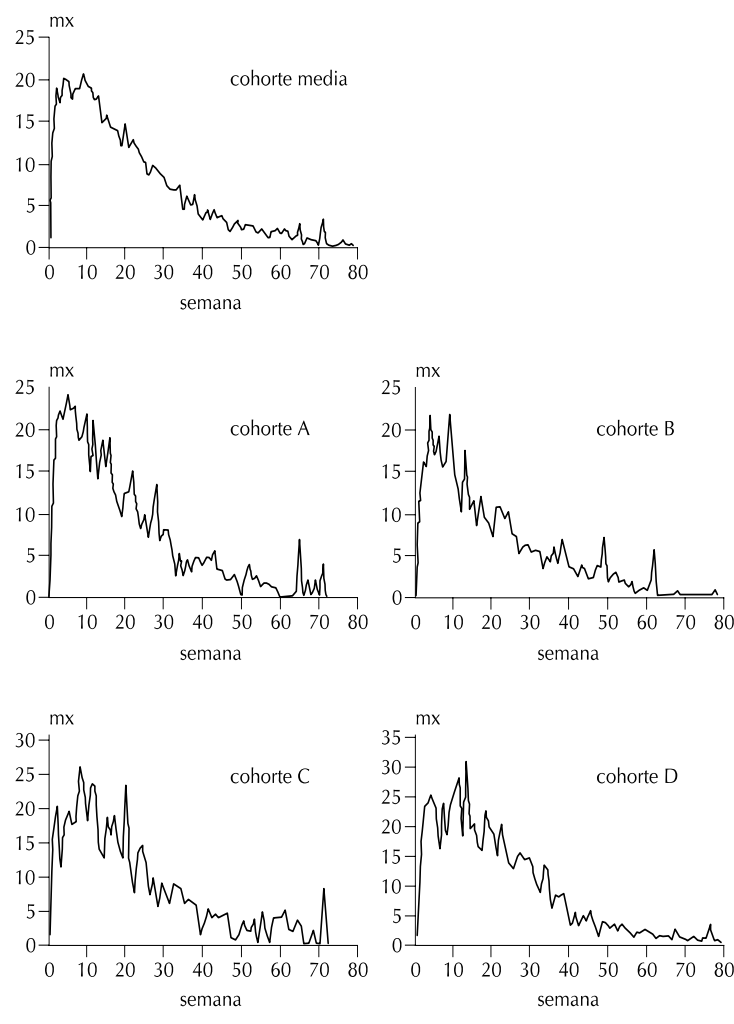

Figura 3 - Fecundidad específica promedio y discriminada según cohorte de Triatoma sordida.

En la Figura 3 se registraron los valores de $m_{x}$ promedios y los correspondientes a las cuatro cohortes. La cohorte A alcanzó su máximo esfuerzo reproductivo a las 5 semanas de ingresar al estado adulto $\left(\mathrm{m}_{\mathrm{x}}=24,0\right)$, oviponiendo durante 31 semanas un número de huevos superior al número promedio de la cohorte $\overline{(X}=6,3)$; la cohorte $B$ mostró 2 picos, uno en la cuarta semana $\left(\mathrm{m}_{\mathrm{x}}=17,2\right)$ y otro en la novena $\left(\mathrm{m}_{\mathrm{x}}=17,3\right)$, con una ovipostura superior a la media $(\bar{X}=3,4)$ durante 4 semanas.

En la octava semana $\left(\mathrm{m}_{\mathrm{x}}=25,3\right)$, la cohorte $\mathrm{C}$ obtuvo su máximo esfuerzo y durante 34 semanas se registraron valores más altos que el promedio $\overline{\mathrm{X}}=6,6)$. La cohorte $\mathrm{D}$ alcanzó en la decimotercer semana el máximo esfuerzo reproductivo $\left(\mathrm{m}_{\mathrm{x}}=30,1\right)$, que representa el mayor valor registrado para todas las cohortes y durante 38 semanas ovipuso un número de huevos por encima del valor medio $\bar{X}=5,6)$.

La curva de fecundidad media de las cuatro cohortes se inicia con valores altos desde la primera semana reproductiva, alcanza la máxima $\mathrm{m}_{\mathrm{x}}$ a las 9 semanas y muestra un último incremento (aunque por debajo del valor promedio) antes de que cese la oviposición (Figura 3).

$\mathrm{Al}$ ajustar los valores a un modelo de regresión se aprecia que después de producido el pico de fecundidad, ésta decrece en proporción constante $\left(\mathrm{m}_{\mathrm{x}}=115,84-24,76 \ln \mathrm{x} ; \mathrm{r}=0,91 ; \mathrm{p}<1 \times 10^{-5}\right)$.

La edad de la última reproducción se registró en las cohortes A, B, C y D a las 71, 78, 71 y 96 semanas respectivamente de haber entrado al estado adulto $\overline{\mathrm{X}}=79$ semanas; $\mathrm{S}=11,8 ; \mathrm{CV}=14,9 \%)$.

\section{Estadísticos Poblacionales}

El tiempo entre generaciones abarcó 61,7 semanas. La media de las tasas de reproducción bruta, instantáneas de natalidad y mortalidad fue: 564,1; 0,086 y 0,005 respectivamente. La tasa de reemplazo fue de 144,3 y la tasa intrínseca de incremento natural fue $0,0824(\lambda=1,085)$. En la cohorte $\mathrm{D}$ se observaron los valores más altos de estos tres últimos parámetros y en la B los más bajos (Tabla 2).

La distribución del valor reproductivo calculado para cada clase de edad fue de tipo normal, con un crecimiento gradual en las edades prereproductivas y una declinación progresiva después de haber alcanzado el pico en una etapa temprana del período reproductor (Tabla 3). Cabe acotar que a la tercer

Tabla 2 - Estadísticas poblacionales de triatoma sordida bajo condiciones controladas de temperatura $\left.28^{\circ} \mathrm{C} \pm 3^{\circ} \mathrm{C}\right)$ y humedad relativa $(63 \% \pm 10 \%)$.

\begin{tabular}{lcccccc}
\hline \multirow{2}{*}{ Estadísticas } & \multicolumn{4}{c}{ Cohortes } \\
\cline { 2 - 6 } & $\mathrm{A}$ & $\mathrm{B}$ & $\mathrm{C}$ & $\mathrm{D}$ & $\mathrm{X}$ & $\mathrm{S}$ \\
\hline Tiempo generacional (semanas) & 59,5 & 70,4 & 55,2 & 60,7 & 61,4 & 6,4 \\
Tasa reproductiva neta & 112,4 & 81,1 & 128,2 & 251,6 & 143,3 & 74,8 \\
Tasa reproductiva bruta & 562,4 & 369,5 & 599,3 & 725,3 & 564,1 & 147,3 \\
Tasa intrínseca de inc. natural & 0,079 & 0,062 & 0,088 & 0,093 & 0,082 & 0,01 \\
Tasa finita de incremento & 1,082 & 1,064 & 1,092 & 1,097 & 1,085 & 0,01 \\
Tasa instantânea de natalidad & 0,087 & 0,066 & 0,092 & 0,099 & 0,086 & 0,01 \\
Tasa instantânea de mortalidad & 0,008 & 0,004 & 0,004 & 0,006 & 0,005 & 0,002 \\
\hline
\end{tabular}


Tabla 3 - Valor reproductivo de Triatoma sordida por estado de desarrollo (al ingresar a cada edad) bajo condiciones controladas de temperatura $\left(28^{\circ} \mathrm{C} \pm 3^{\circ} \mathrm{C}\right)$ y humedad relativa $(63 \% \pm 10 \%)$.

\begin{tabular}{lrrrrrr}
\hline \multirow{2}{*}{ Estadio } & \multicolumn{7}{c}{ Cohortes } \\
\cline { 2 - 7 } & $\mathrm{B}$ & $\mathrm{C}$ & $\mathrm{D}$ & $\mathrm{X}$ & $\mathrm{S}$ & \\
\hline Huevo & 1,4 & 1,6 & 1,6 & 1,5 & 1,5 & 0,09 \\
N1 & 1,6 & 1,7 & 1,9 & 2,0 & 1,8 & 0,18 \\
N2 & 2,3 & 2,1 & 2,8 & 2,9 & 2,5 & 0,39 \\
N3 & 3,6 & 2,6 & 4,3 & 4,3 & 3,7 & 0,80 \\
N4 & 6,7 & 4,5 & 8,3 & 8,4 & 7,0 & 1,82 \\
N5 & 15,6 & 9,4 & 16,6 & 16,3 & 14,5 & 3,41 \\
Adultos & 96,0 & 41,8 & 79,8 & 122,5 & 85,0 & 33,8 \\
Vx Total & $3.248,2$ & $1.635,4$ & $3.148,6$ & $4.689,6$ & $3.180,4$ & $1.247,7$ \\
Vx Maximo & 108,3 & 50,4 & 93,6 & 136,9 & 97,3 & 36,1 \\
\hline
\end{tabular}

semana de haber ingresado al estado adulto, se verificó el máximo $\mathrm{V}_{\mathrm{x}}$ en las cohortes $\mathrm{A}$ y $\mathrm{D}$, mientras las hembras de las cohortes $\mathrm{B}$ y $\mathrm{C}$ obtuvieron su máximo $\mathrm{V}_{\mathrm{x}}$ recién en la cuarta y quinta semana de vida como adultos, respectivamente. Se calculó que, de producirse una distribución estable de edades, una población de T. sordida estaría compuesta por $32 \%$ de huevos, $26 \%$ de ninfas de primer estadio, $19 \%$ de ninfas de segundo estadio, $13 \%$ de ninfas de tercer estadio, $6 \%$ de ninfas de cuarto estadio, $3 \%$ de ninfas de quinto estadio y $1 \%$ de adultos.

\section{DISCUSIÓN}

La curva de supervivencia observada se aproxima al tipo II $^{15}$ debido a que un número aproximadamente constante de individuos muere en cada edad. Sin embargo, pueden citarse algunas excepciones, como la registrada en la semana previa a la edad media de ingreso al estado adulto, en la cual murieron un elevado número de individuos. La expectativa de vida fue declinando en forma gradual, sin registrarse picos importantes.

A diferencia de lo verificado en T. sordida, en $T$. infestans se observa un patrón de mortalidad menos definido ${ }^{13}$.

La expectativa de vida de los machos de T. sordi$d a$ fue similar a la de las hembras, en coincidencia a lo obtenido con T. guasayana ${ }^{9}$ y T. breyeri ${ }^{10}$ y difiere de $T$. infestans ${ }^{13}$, especie en la que los machos superan en su $\mathrm{e}_{\mathrm{x}}$ a las hembras.

Respecto al comportamiento reproductor de las hembras de $T$. sordida, se comprueba que, en promedio, a las 9 semanas de ingresar al estado adulto alcanzan su máxima fecundidad. Este valor es próximo al de $T$. infestans $\left(10,5\right.$ semanas $\left.^{13}\right)$ y superior al de $T$. breyeri $\left(4,67\right.$ semanas $\left.^{10}\right)$ y al de $T$. guasayana (3,3 semanas $\left.^{9}\right)$.

El tiempo necesario para que se complete una generación de T. sordida es similar al de T. guasayana $\left(57,9\right.$ semanas $\left.^{9}\right)$ y $T$. breyeri $\left(58,1\right.$ semanas $\left.^{10}\right)$ y mayor que el requerido por $T$. infestans $(30,8$ se$\operatorname{manas}^{13}$ ).

La tasa de reemplazo de $T$. sordida es alta si se la compara con las de T. infestans $\left(25,04^{13}\right)$, T. guasaya$n a\left(8,08^{9}\right)$ y $T$. breyeri $\left(2,68^{10}\right)$. Por otra parte, una característica reproductiva tal como la tasa intrínseca de incremento natural es más próxima a la de $T$. infestans $\left(\mathrm{r}=0,101^{13}\right)$ que a la de $T$. guasayana $\left(\mathrm{r}=0,036^{9}\right)$ y $T$. breyeri $\left(\mathrm{r}=0,017^{10}\right)$.

Al igual que en T. infestans ${ }^{13}$, se comprueba en $T$. sordida que el $\mathrm{V}_{\mathrm{x}}$ aumenta con la edad hasta llegar al máximo en las primeras semanas de la edad reproductiva.

Las diferentes condiciones experimentales utilizadas por Rabinovich* y por Ghilini**, así como las diferencias interespecíficas podrían explicar la divergencia entre los resultados obtenidos por los citados autores y los del presente trabajo.

Si la población de T. sordida llegara a un punto de equilibrio, la distribución de edades resultante sería semejante a la calculada para $T$. infestans ${ }^{13}$ con un mayor porcentaje de ninfas $(67 \%)$ que de huevos $(32 \%)$ y adultos $(1 \%)$.

En condiciones naturales, las poblaciones de $T$. sordida capturadas estaban compuestas por un alto porcentaje de ninfas y un mínimo de huevos. Por ejemplo, la estructura de edad correspondiente a los ejemplares colectados en un palomar del Parque Mitre (Corrientes, Mayo de 1984) estaba constituida por un $0,3 \%$ de huevos, $96,2 \%$ de ninfas y $3,5 \%$ de adultos $^{2}$, y la población de un gallinero del área rural de San Luis del Palmar (Corrientes, Argentina, Junio

* criadas a $26^{\circ} \mathrm{C} \pm 1^{\circ} \mathrm{C}-60 \% \pm 10 \%$. Alimentación : gallinas $/ 1 \mathrm{~h}$.

** criadas a $25^{\circ} \mathrm{C} \pm 4{ }^{\circ} \mathrm{C}-60 \% \pm 20 \%$. Alimentación : ratas. 
de 1986), estaba integrada por un $1 \%$ de huevos, $92 \%$ de ninfas y $0,7 \%$ de adultos ${ }^{1}$.

Las diferencias con la estructura de edad calculada podría deberse a la época del año en que fueron realizados los censos, a la dificultad de obtener durante los muestreos la totalidad de los ejemplares y a que en la naturaleza es poco probable que una población mantenga una estructura estable de edades, como ha sido probado en las poblaciones de T. sordida, las que presentan variaciones estacionales en su conformación ${ }^{3,7}$.

En condiciones experimentales, una población de T. sordida presenta las siguientes características: a) Dilatado período preimaginal, que se traduce en una

\section{REFERENCIAS BIBLIOGRÁFICAS}

1. BAR, M.E.; OSCHEROV, E.B.; DAMBORSKY, M.P.; VARELA, M.E.; MIZDRAJI, G.; PORCEL, E. Triatomismo del Departamento San Luis del Palmar de la Provincia de Corrientes, Argentina. Medicina (B. Aires), 52: 193-201, 1992.

2. BAR, M.E.; OSCHEROV, E.B.; DAMBORSKY, M.P. Presencia de Triatoma sordida Stål, 1859 en ecótopos urbanos de la ciudad de Corrientes, Argentina. Rev. Saúde Pública, 27: 117-22, 1993.

3. DIOTAIUTI, L.; LOIOLA, C. F.; FALCÃO, P. L.; DIAS, J. C. P. The ecology of Triatoma sordida in natural environments in two different regions of the State of Minas Gerais, Brazil. Rev. Inst. Med. Trop. S. Paulo, 35: 237-45, 1993.

4. FORATTINI, O. P.; ROCHA E SILVA, E.; FERREIRA, O.; RABELLO, E. X.; PATTOLI, D.G.B. Aspectos ecológicos da tripanossomiase americana. III. Dispersão local de triatomineos com especial referência ao Triatoma sordida. Rev. Saúde Pública, 5: 193-205, 1971.

5. FORATTINI, O.P.;. BARATA, J. M. S.; SANTOS, J. L. F.; SILVEIRA, A.C. Hábitos alimentares, infecção natural e distribução de triatomineos domiciliados na região nordeste do Brasil. Rev. Saúde Púbica, 15: 3-64, 1981.

6. FORATTINI, O.P.; BARATA, J. M. S.; SANTOS J. L. F.; SILVEIRA, A.C. Hábitos alimentares, infecção natural e distribução de triatomineos domiciliados na região central do Brasil. Rev. Saúde Pública, 16: 171-204, 1982.

7. FORATTINI, O. P.; FERREIRA,O. A.; RABELLO, E. X.; BARATA, J. M. S.; SANTOS, J. L. F. Aspectos ecológicos da tripanossomiase americana. XVIII- Desenvolvimento e ciclos anuais de colônias de Triatoma infestans, Triatoma sordida e Rhodnius neglectus em ecotopos artificiais, no ambiente peri e extradomiciliar. Rev. Saúde Pública., 17: 243-62, 1983. reproducción tardía, b) curva de supervivencia de tipo II ${ }^{15}$, c) prolongada supervivencia de los adultos, d) tiempo generacional mayor a un año, e) gran capacidad de ayuno ${ }^{11}$, f) reproducción reiterada, g) tasa de incremento natural baja. Por lo antes expuesto, $T$. sordida se comportaría como una especie en equilibrio $^{12}$ al adoptar una estrategia próxima a la $\mathrm{K}$; característica que la hace semejante a T. infestans ${ }^{13}$.

\section{AGRADECIMIENTOS}

A los Doctores Arturo Kher y Ricardo Gürtler por sus valiosas críticas y sugerencias.

8. FREITAS, J. L. P.; SIQUEIRA, A. F.; FERREIRA, O. A. Investigações epidemiológicas sobre triatomineos de hábitos domésticos e silvestres com o auxilio da reação de precipitina. Rev. Inst. Med. Trop. S. Paulo, 2: 90-9, 1960.

9. GHILINI, J.M. Estadísticas vitales de Triatoma guasayana Wygodzinsky y Abalos, 1949 (Hemiptera : Reduviidae) bajo condiciones controladas de laboratorio. Rev. Soc. Entomol. Argent., 41: 211-24, 1982.

10. GHILINI, J.M. Estadísticas vitales de Triatoma breyeri Del Ponte, 1929 (Hemiptera : Reduviidae) bajo condiciones de laboratorio. Rev. Soc. Entomol. Argent., 42: 101-11, 1983.

11. JUAREZ, E. \& CASTRO SILVA, E.P. de. Comportamento do Triatoma sordida em condições de laboratório. Rev. Saúde Pública, 16 (supl.): 1-36, 1982.

12. PIANKA, E.R. Ecología evolutiva. Barcelona, Omega, 1982.

13. RABINOVICH, J.E. Vital statistics of Triatominae (Hemiptera : Reduviidae) under laboratory conditions. I. Triatoma infestans (Klug). J. Med. Entomol., 9: 351-70, 1972.

14. ROCHA E SILVA, E.O.; SOUZA, J.M. P. de; ANDRADE, J. C. R. de; MELLO, C. da S.; FERREIRA, O. A. Preferência alimentar (entre sangue humano e ave) dos Triatoma sordida encontrados em casas habitadas da região norte do Estado de São Paulo, Brasil. Rev. Saúde Pública, 11: 258-69, 1977.

15. SLOBODKIN, L.B. Crecimiento y regulación de las poblaciones animales. Buenos Aires, EUDEBA. 1966.

16. WORLD HEALTH ORGANIZATION. Control of Chagas Disease. Geneva, 1991. (WHO, Technical Report Series, 811). 